\title{
The neutrophil to lymphocyte ratio in heart failure: a comprehensive review
}

\author{
CATERINA DELCEA ${ }^{1,2,3}$, CĂTĂLIN ADRIAN BUZEA $^{1,2}$, GHEORGHE ANDREI DAN $^{1,2,3}$ \\ ${ }^{1}$ Carol Davila University of Medicine and Pharmacy, Bucharest, Romania \\ ${ }^{2}$ Cardiology Department, Colentina Clinical Hospital, Bucharest, Romania \\ ${ }^{3}$ CDPC Cardiology Laboratory, Colentina Clinical Hospital, Bucharest, Romania
}

\begin{abstract}
Introduction. Heart failure (HF) and systemic inflammation are interdependent processes that continuously potentiate each other. Distinct pathophysiological pathways are activated, resulting in increased neutrophil count and reduced lymphocyte numbers, making the neutrophil to lymphocyte ratio (NLR) a potential indirect marker of severity. We conducted this comprehensive review to characterize the role of NLR in HF.

Methods. We searched the PubMed (MEDLINE) database using the key words "neutrophil", "lymphocyte", "heart failure", "cardiomyopathy", "implantable cardioverter defibrillator", "cardiac resynchronization therapy" and "heart transplant".

Results. We identified 241 publications. 31 were selected for this review, including 12,107 patients. NLR was correlated to HF severity expressed by clinical, biological, and imaging parameters, as well as to short and long-term prognosis. Most studies reported its survival predictive value. Elevated NLR (>2.1-7.6) was an independent predictor of in-hospital mortality [adjusted HR $1.13(95 \%$ CI 1.01-1.27) - 2.8 (95\% CI 1.43-5.53)] as well as long-term all-cause mortality [adjusted HR 1.43 (95\% CI 1.1-1.85) - 2.403 (95\% CI 1.076-5.704)].

Higher NLR levels also predicted poor functional capacity [NLR $>2.26 / 2.74$, HR $3.93(95 \%$ CI 1.02-15.12) / 3.085 (95\% CI 1.52-6.26)], hospital readmissions [NLR > 2.9/7.6, HR 1.46 (95\% CI $1.10-1.93) / 3.46(95 \%$ CI 2.11-5.68)] cardiac resynchronization therapy efficacy [NLR $>3.45 /$ unit increase, HR 12.22 (95\% CI 2.16-69.05) / 1.51 (95\% CI 1.01-2.24)] and appropriate implantable cardioverter defibrillator shocks (NLR > 2.93), as well as mortality after left ventricular assist device implantation [NLR > 4.4 / quartiles, HR 1.67 (95\% CI 1.03-2.70) / 1.22 (95\% CI 1.01-1.47)] or heart transplant (NLR > 2.41, HR 3.403 (95\% CI 1.04-11.14)].

Conclusion. Increased NLR in HF patients can be a valuable auxiliary biomarker of severity, and most of all, of poor prognosis.
\end{abstract}

Key words: neutrophil to lymphocyte ratio, NLR, heart failure, cardiomyopathy, survival prognosis.

\section{INTRODUCTION}

Heart failure (HF) and systemic inflammation are two interlinked, continuously evolving, and coexisting processes that constantly emphasize each other [1,2]. The initial cytokine hypothesis postulated that endogenous cytokines induce HF progression [3]. However, more and more data support the reverse theory as well, proving that HF induces inflammation in both the myocardium itself as well as other tissues and organs [4]. Regardless of the HF substrate, its multiple possible etiologies or its coinciding risk factors, inflammation parameters are related to HF prognosis, with worst outcomes in patients with more prevalent and higher expression of inflammatory biomarkers [5].
HF and inflammation influence the leukocyte homeostasis. Neutrophils are highly susceptible to inflammation not only in infection, but also in heart failure, where an increased lifespan was observed due to delayed apoptosis [6]. Modulating signaling pathways by myeloperoxidase release, for example, they lead to tissue and endothelial damage, contributing to myocardial remodeling [7]. Neutrophilia was therefore independently associated to heart failure severity and prognosis $[8,9]$.

Systemic release of cytokines induced either by the severity of congestion or by episodes of acute decompensation may lead to lymphocyte apoptosis, while higher levels of cortisol and catecholamines secondary to physiological stress may also play an important part in the lymphocytes' number and 
function $[10,11]$. Considered another marker of inflammation and severity in HF, lymphopenia was also independently correlated to poor NYHA class [12] and mortality $[13,14]$.

Consequently, integrating different yet interdependent immune pathways, the neutrophil to lymphocyte ratio (NLR) was independently correlated to prognosis in various cardiovascular diseases [15], predominantly ischemic heart disease [16], but also arrhythmias [17], valvular disease [18] and heart failure [18].

The purpose of this review was to assess the utility of NLR in HF patients.

\section{MATERIAL AND METHODS}

\section{Search strategy}

We initially searched the PubMed database from its launch to February $1^{\text {st }} 2019$ using the key words "neutrophil", "lymphocyte", "heart failure", "cardiomyopathy", "implantable cardioverter defibrillator", "cardiac resynchronization therapy" and "heart transplant". A second search was conducted on May $1^{\text {st }} 2019$ using the same strategy and these results were considered final. The strategy is detailed in Table 2 Supplemental material.

Only articles written in English were evaluated.

References of all included studies as well as excluded reviews or letters to the editor were screened for inclusion.

Two authors independently reviewed the articles obtained from the literature search and selected the ones to be included in the analysis. In case of disagreement, a third author decided the inclusion or exclusion of articles.

\section{Inclusion criteria}

\section{Types of studies}

Prospective as well as retrospective observational or descriptive studies that analyzed the neutrophil lymphocyte ratio in adult heart failure patients were evaluated.

\section{Types of participants}

In order to be considered for the review, the studies had to have a clear description of the selected study sample and to mention that the patients had heart failure.

We applied no restriction regarding sample size, study duration, or interventions administered to the patients.

\section{Exclusion criteria}

Case reports, reviews, and letters to the editor were excluded. Studies including children or adolescents were excluded. Studies that had no mention of heart failure as a baseline characteristic of patients were excluded. Studies that analyzed populations with other baseline cardiovascular diseases, such as ischemic heart disease or arterial hypertension, with only part of the participants having heart failure were excluded. Studies with no separate analysis for heart failure patients were excluded. Studies about valvular replacement procedures with no mention of heart failure were excluded. Since the baseline characteristics of patients did not include HF, studies where NLR was used as a predictor for developing HF were excluded.

\section{Outcomes}

We applied no restrictions in regard to reported outcomes, therefore we included studies describing associations of NLR with parameters of heart failure severity, comorbidities, or biomarkers, as well as studies demonstrating the predictive value of NLR for short or long-term mortality, hospital readmissions, major cardiovascular adverse events, poor functional capacity, response to cardiac resynchronization therapy, adequate implantable cardioverter defibrillator shocks, mortality after left ventricular assist device implantation or after heart transplantation.

\section{RESULTS}

We identified 241 publications. 31 publications fulfilled the inclusion and exclusion criteria and were selected for the review with a total of 12107 patients. The selection process is detailed in Figure 1.

Details and characteristics of all included studies are listed in Table 1. 


\begin{tabular}{|c|c|c|c|c|c|c|c|c|}
\hline & 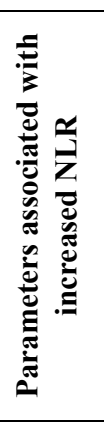 & 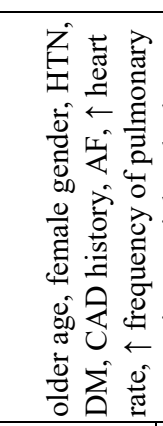 & 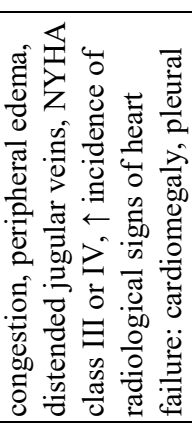 & 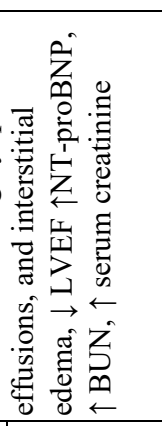 & 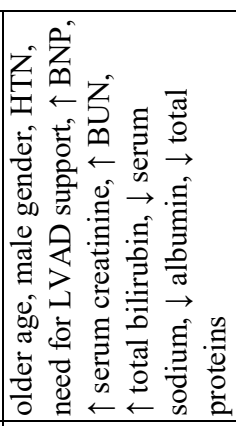 & & & \\
\hline & 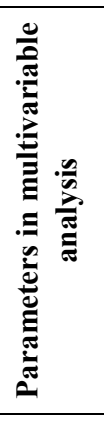 & 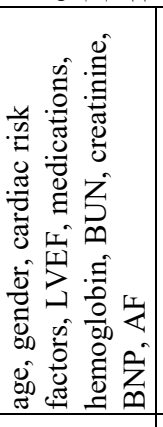 & 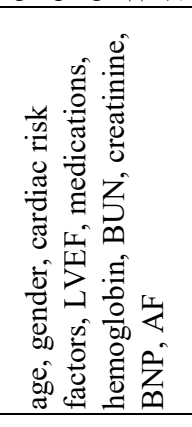 & & & 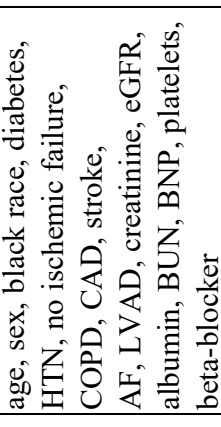 & & 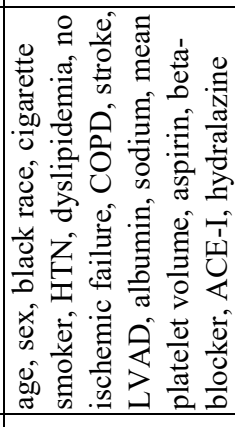 \\
\hline $\begin{array}{l}3 \\
.0 \\
0 \\
0 \\
0 \\
0 \\
\Xi \\
. \Xi \\
0\end{array}$ & 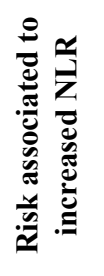 & 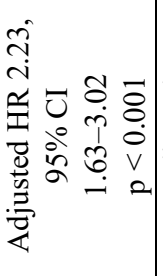 & 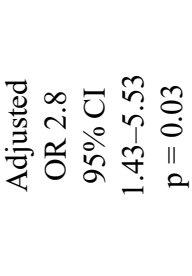 & 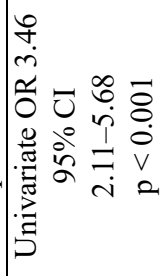 & 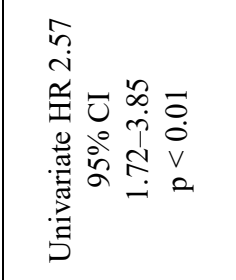 & 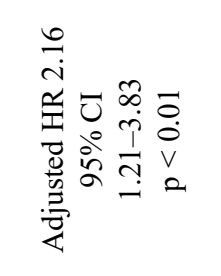 & 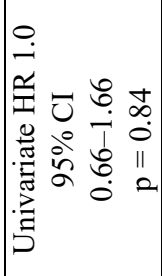 & 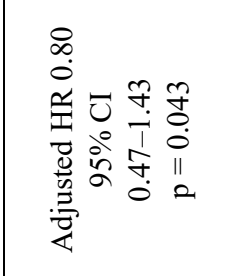 \\
\hline 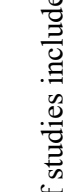 & 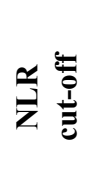 & 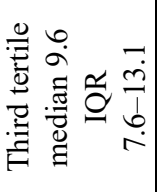 & 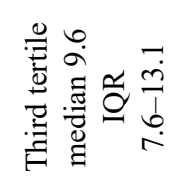 & 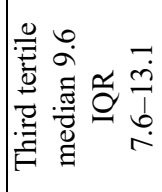 & 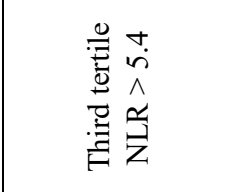 & 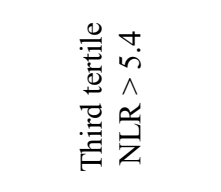 & 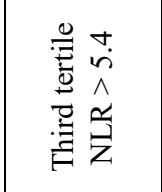 & 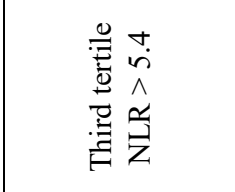 \\
\hline 害 & 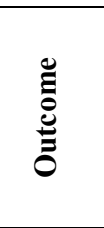 & 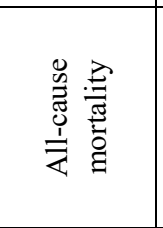 & 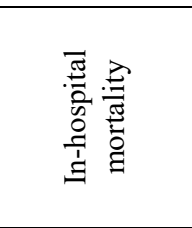 & 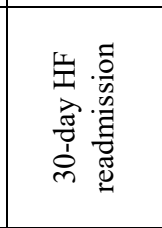 & 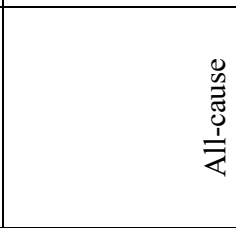 & & & 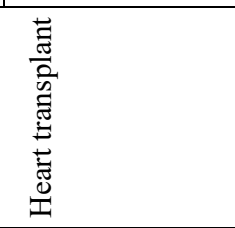 \\
\hline & 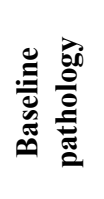 & & 崖 & & & 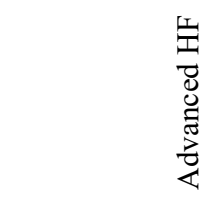 & & \\
\hline & 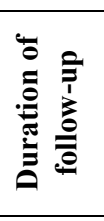 & & 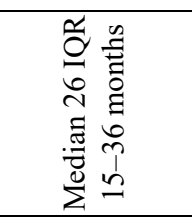 & & & 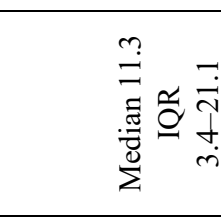 & & \\
\hline & 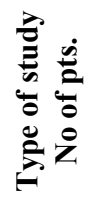 & & 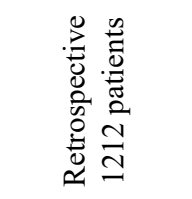 & & & 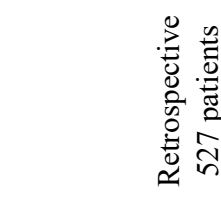 & & \\
\hline & 泀离 & & 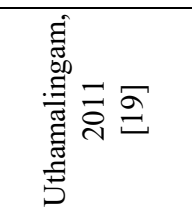 & & & 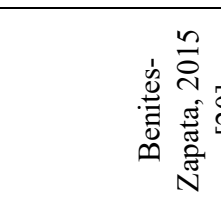 & & \\
\hline
\end{tabular}




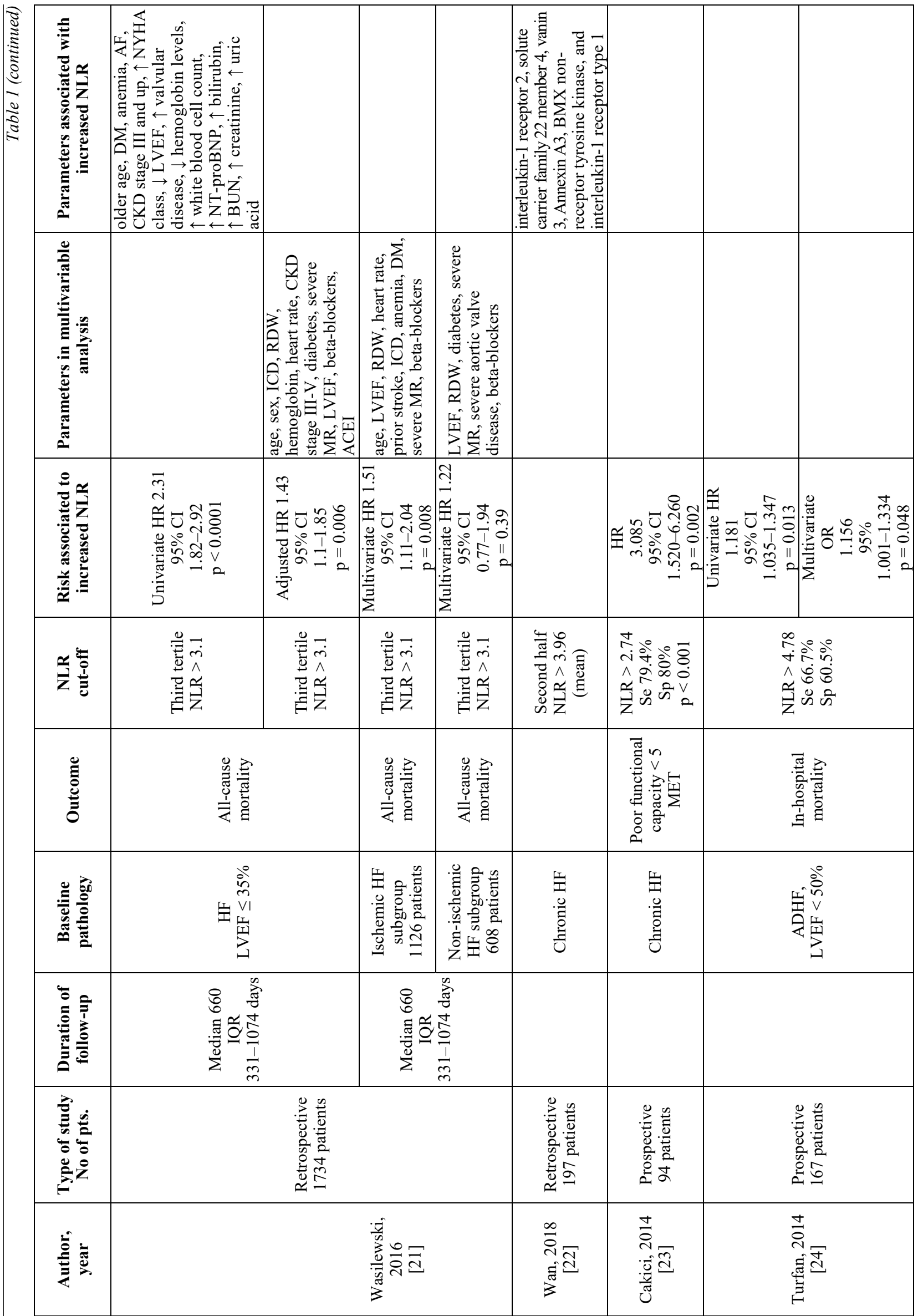




\begin{tabular}{|c|c|c|c|c|c|c|c|}
\hline 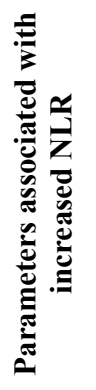 & & & & & & & \\
\hline 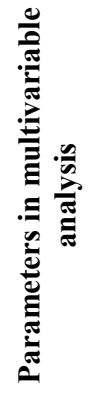 & & & & & & & \\
\hline 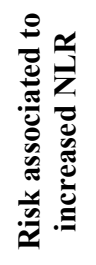 & 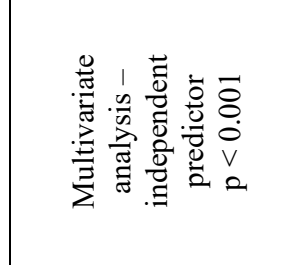 & 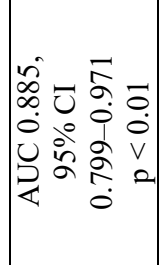 & 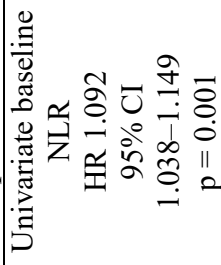 & 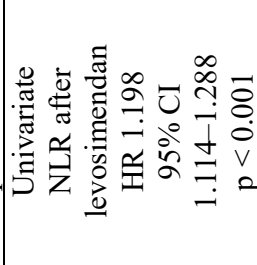 & 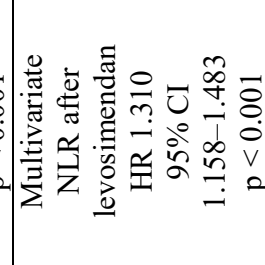 & $\mid$\begin{tabular}{cc}
0 & \\
0 & $n$ \\
0 & 0 \\
0 & 0 \\
0 & 0 \\
0 & 11 \\
\hdashline & 0
\end{tabular} & 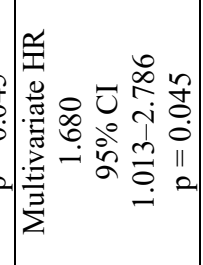 \\
\hline$\frac{\dddot{2}}{z}$ & 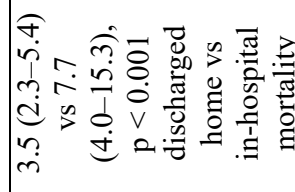 & 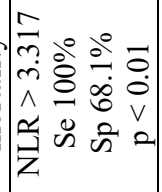 & & 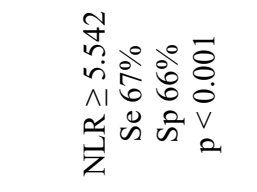 & & & 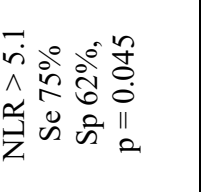 \\
\hline 节 & 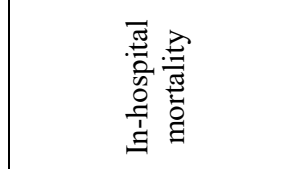 & 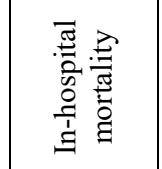 & & 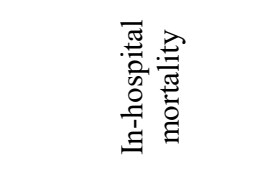 & & & 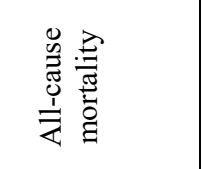 \\
\hline 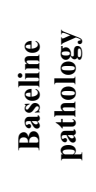 & 崖 & 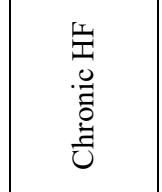 & & 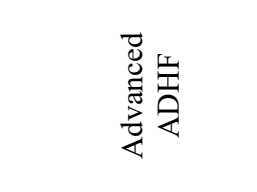 & & & 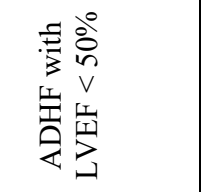 \\
\hline 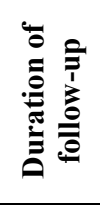 & & 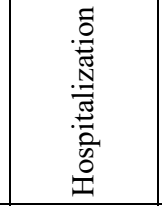 & & 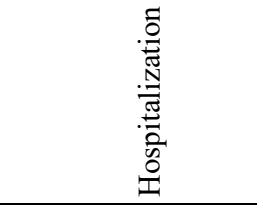 & & & 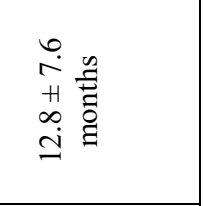 \\
\hline 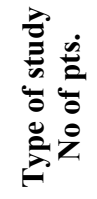 & 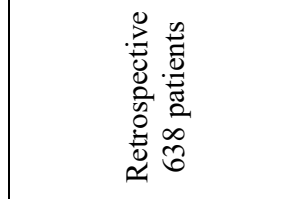 & 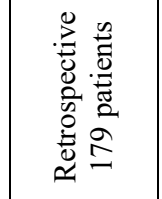 & & 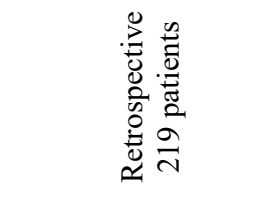 & & & 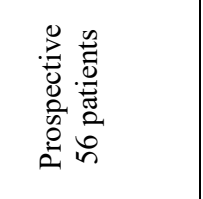 \\
\hline 䒻 & 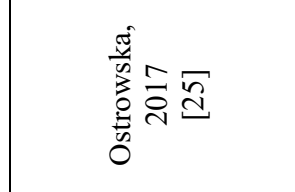 & 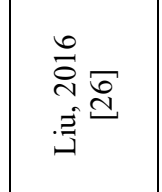 & & 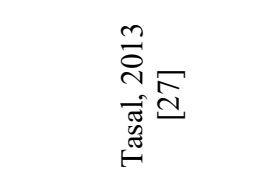 & & & 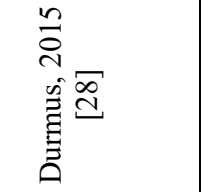 \\
\hline
\end{tabular}




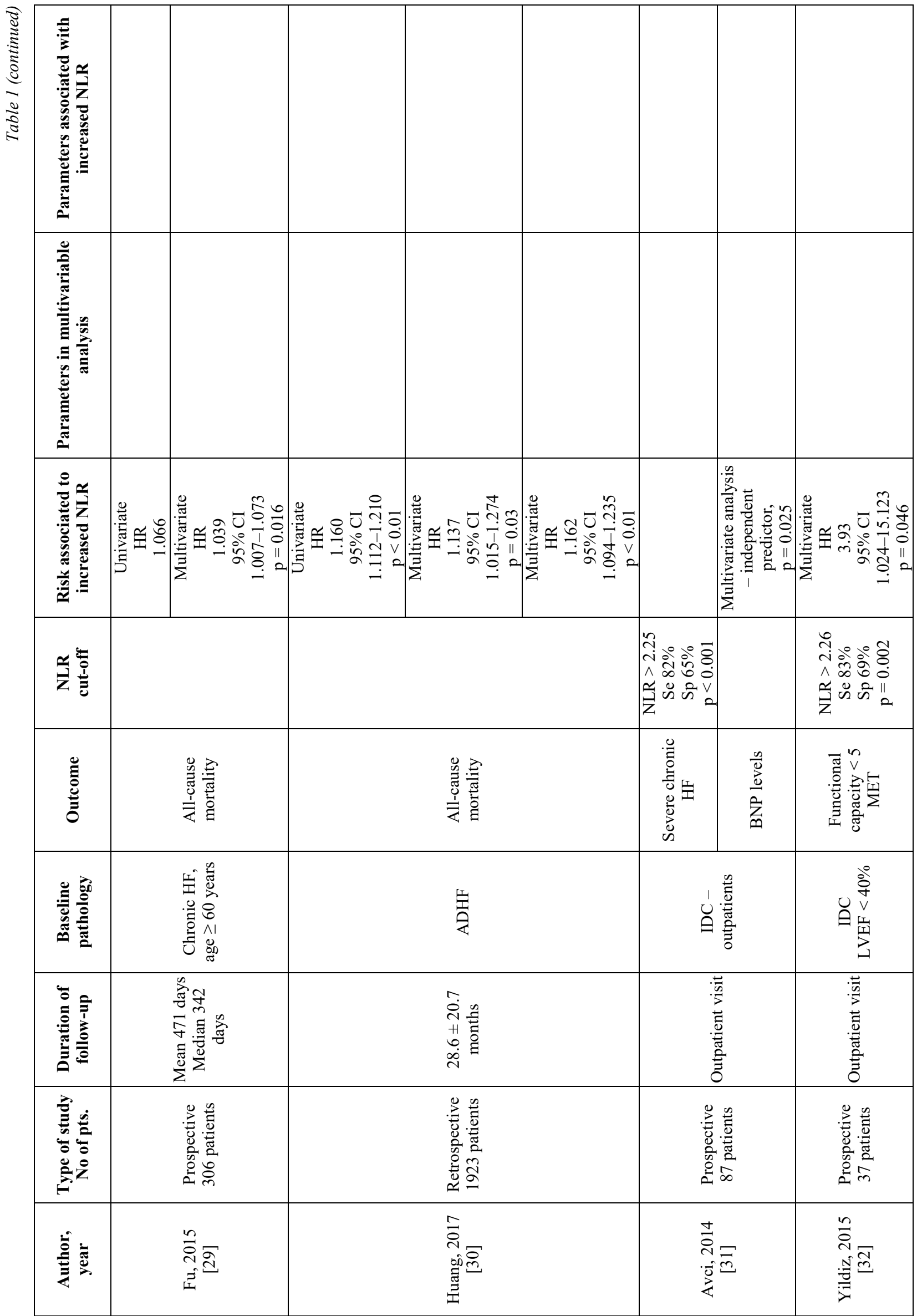




\begin{tabular}{|c|c|c|c|c|c|c|c|c|c|}
\hline 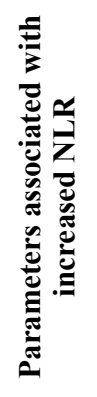 & & & & & & & & & \\
\hline 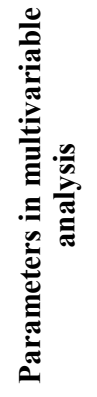 & & & & & & & & & \\
\hline 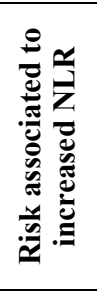 & & 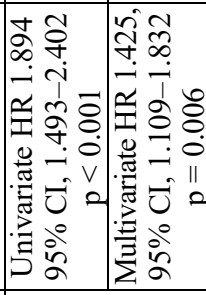 & 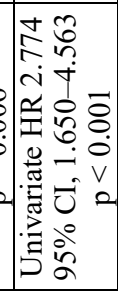 & 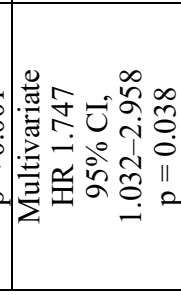 & 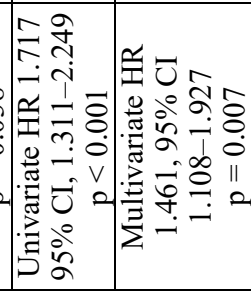 & 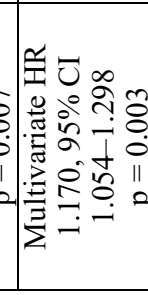 & 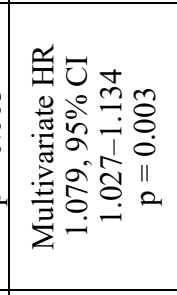 & 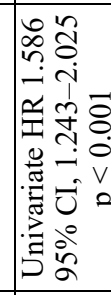 & 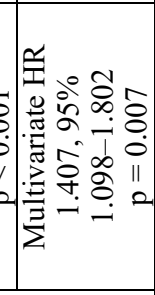 \\
\hline 莫 & 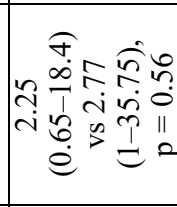 & & & 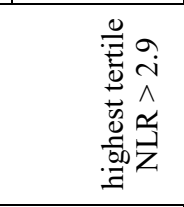 & & & 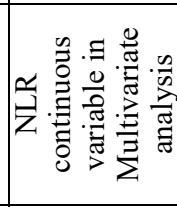 & 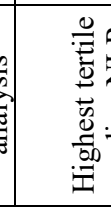 & 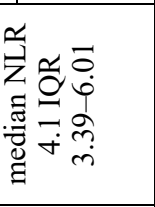 \\
\hline 䓂 & 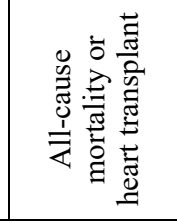 & $\stackrel{\text { U⿱山己心 }}{\Sigma}$ & & 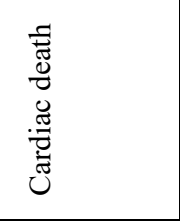 & 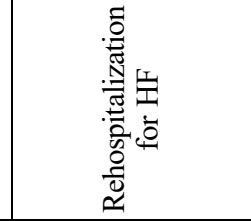 & 党 & 宸 & 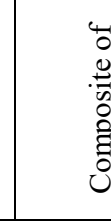 & 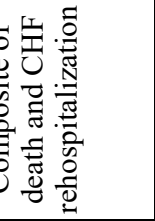 \\
\hline 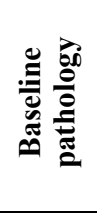 & 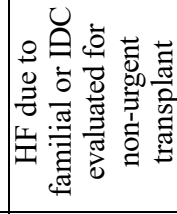 & & & 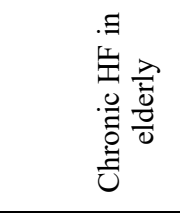 & & & & 岂 & \\
\hline 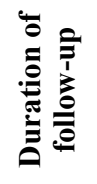 & 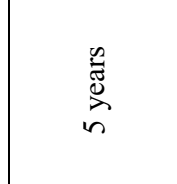 & & & 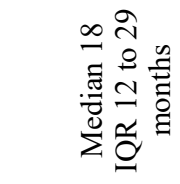 & & & & 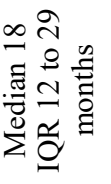 & \\
\hline 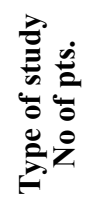 & 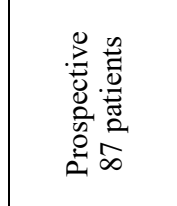 & & & 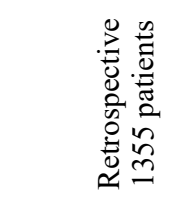 & & & & 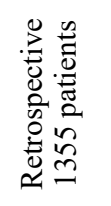 & \\
\hline 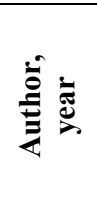 & 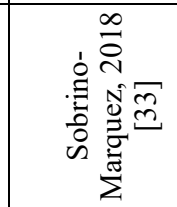 & & & $\begin{array}{l}\stackrel{0}{\vec{*}} \\
\text { हే } \\
\text { है }\end{array}$ & & & & 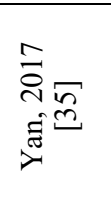 & \\
\hline
\end{tabular}




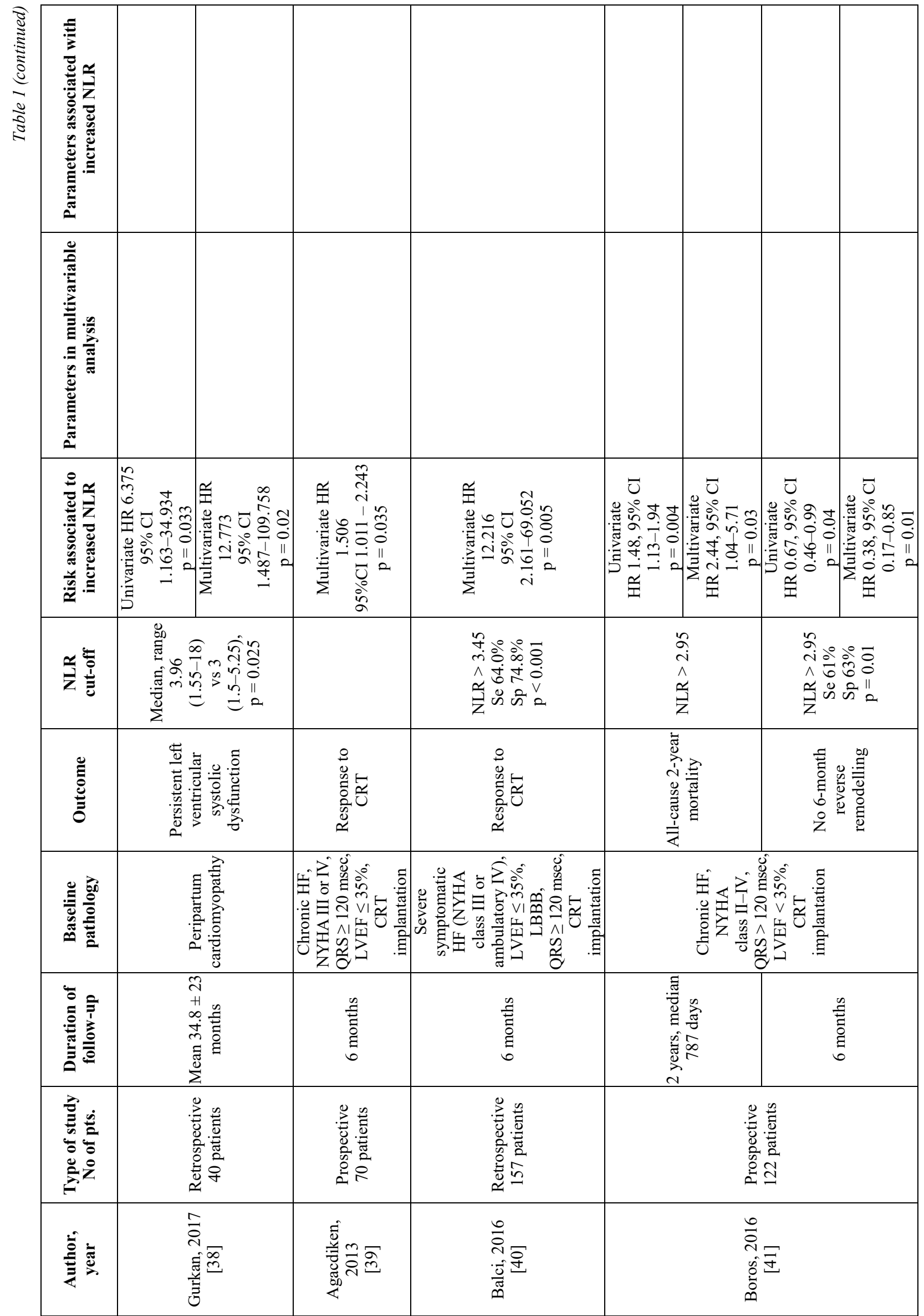




\begin{tabular}{|c|c|c|c|c|c|c|c|}
\hline 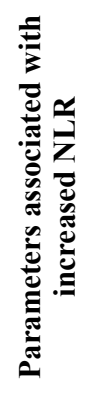 & & & & & & & \\
\hline 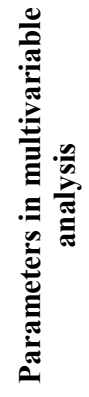 & & & & & & & \\
\hline 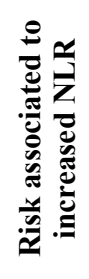 & 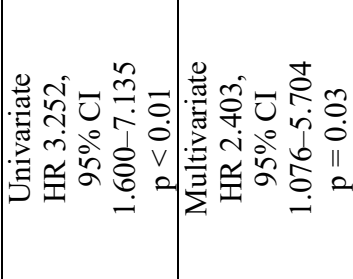 & 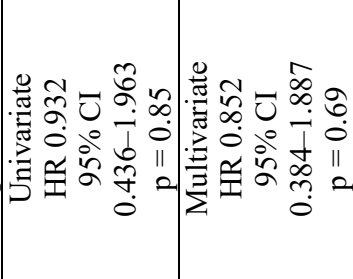 & 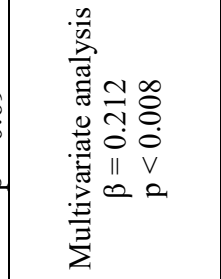 & 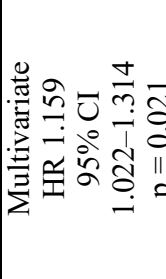 & 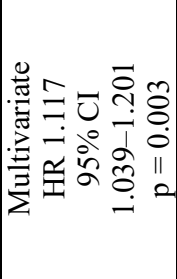 & & \\
\hline 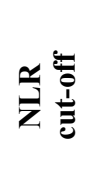 & \multicolumn{2}{|c|}{ 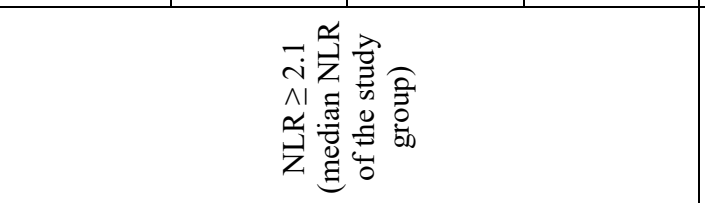 } & 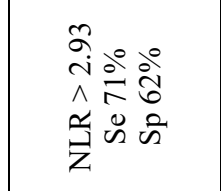 & \multicolumn{2}{|c|}{ 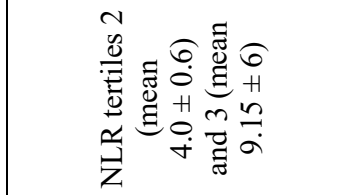 } & 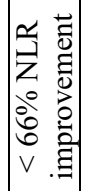 & 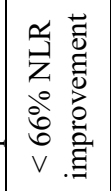 \\
\hline 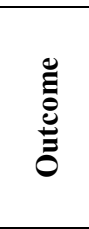 & \multicolumn{2}{|l|}{ 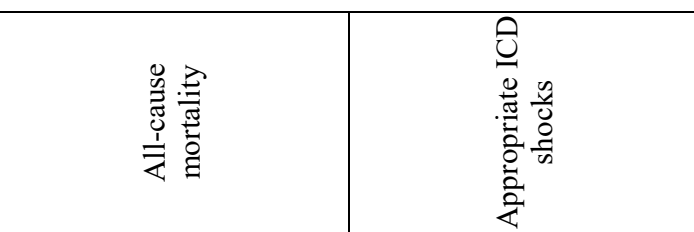 } & 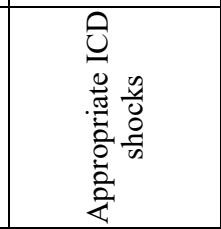 & 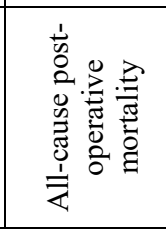 & 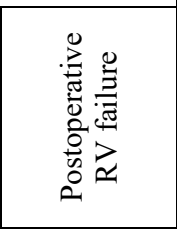 & 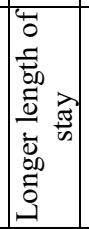 & 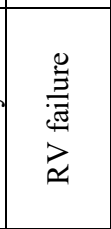 \\
\hline 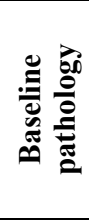 & \multicolumn{2}{|c|}{ 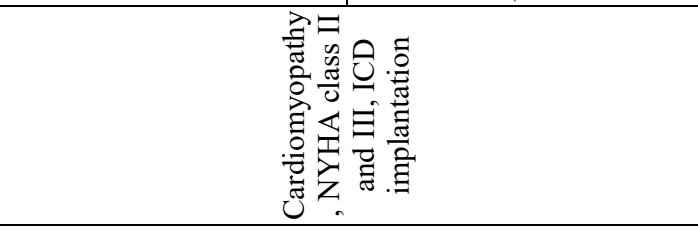 } & 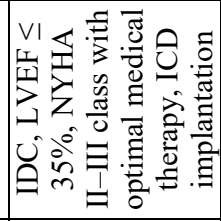 & \multicolumn{2}{|c|}{ 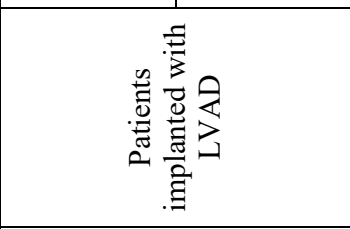 } & \multicolumn{2}{|c|}{ 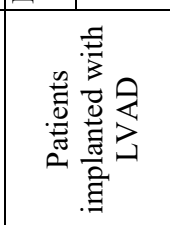 } \\
\hline 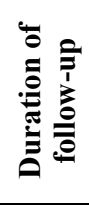 & \multicolumn{2}{|c|}{ 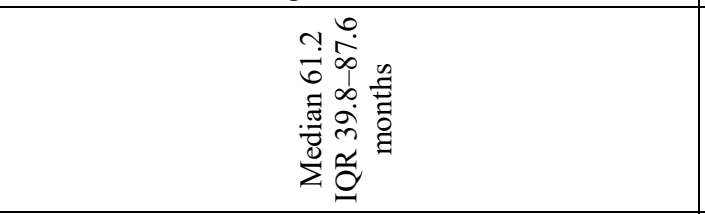 } & 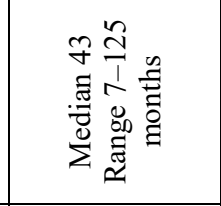 & \multicolumn{2}{|c|}{ 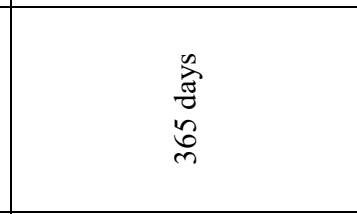 } & \multicolumn{2}{|c|}{ 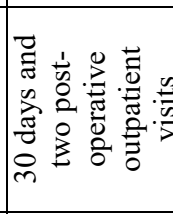 } \\
\hline 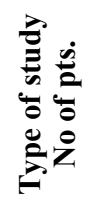 & \multicolumn{2}{|c|}{ 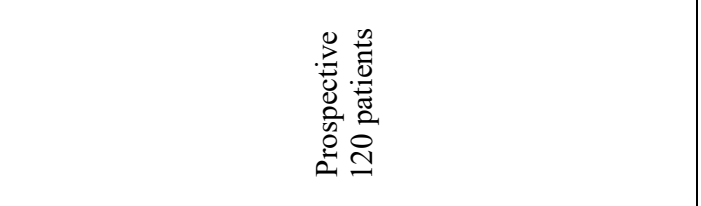 } & 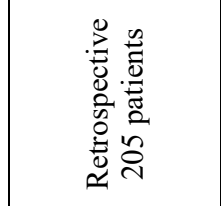 & \multicolumn{2}{|c|}{ 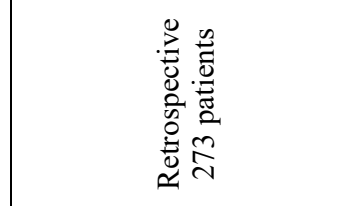 } & \multicolumn{2}{|c|}{ 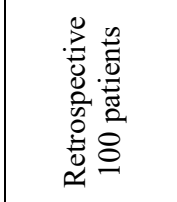 } \\
\hline 泀离 & \multicolumn{2}{|r|}{ 离 } & 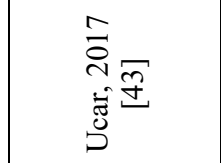 & \multicolumn{2}{|c|}{ 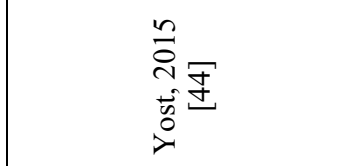 } & \multicolumn{2}{|c|}{ 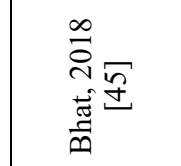 } \\
\hline
\end{tabular}




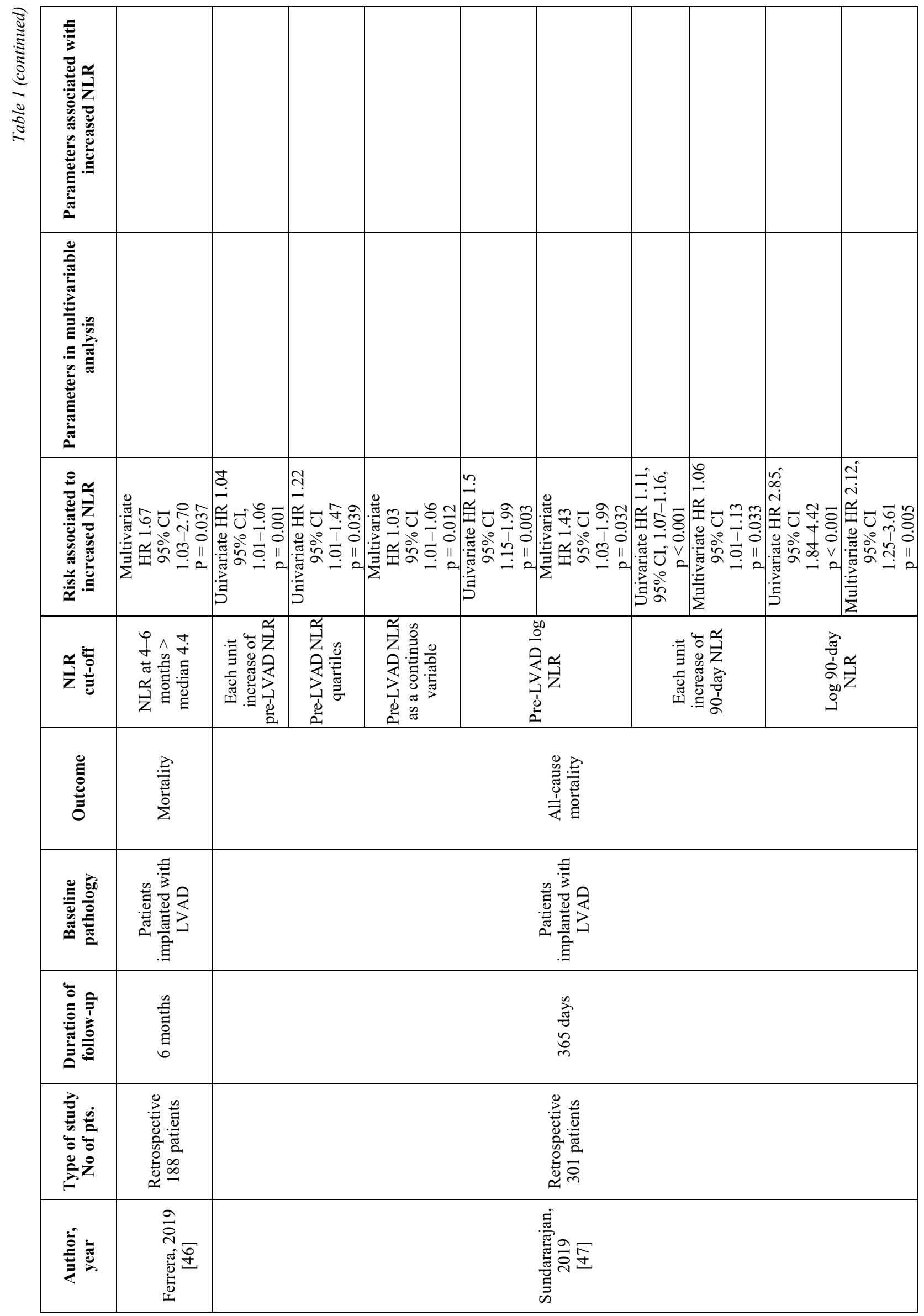




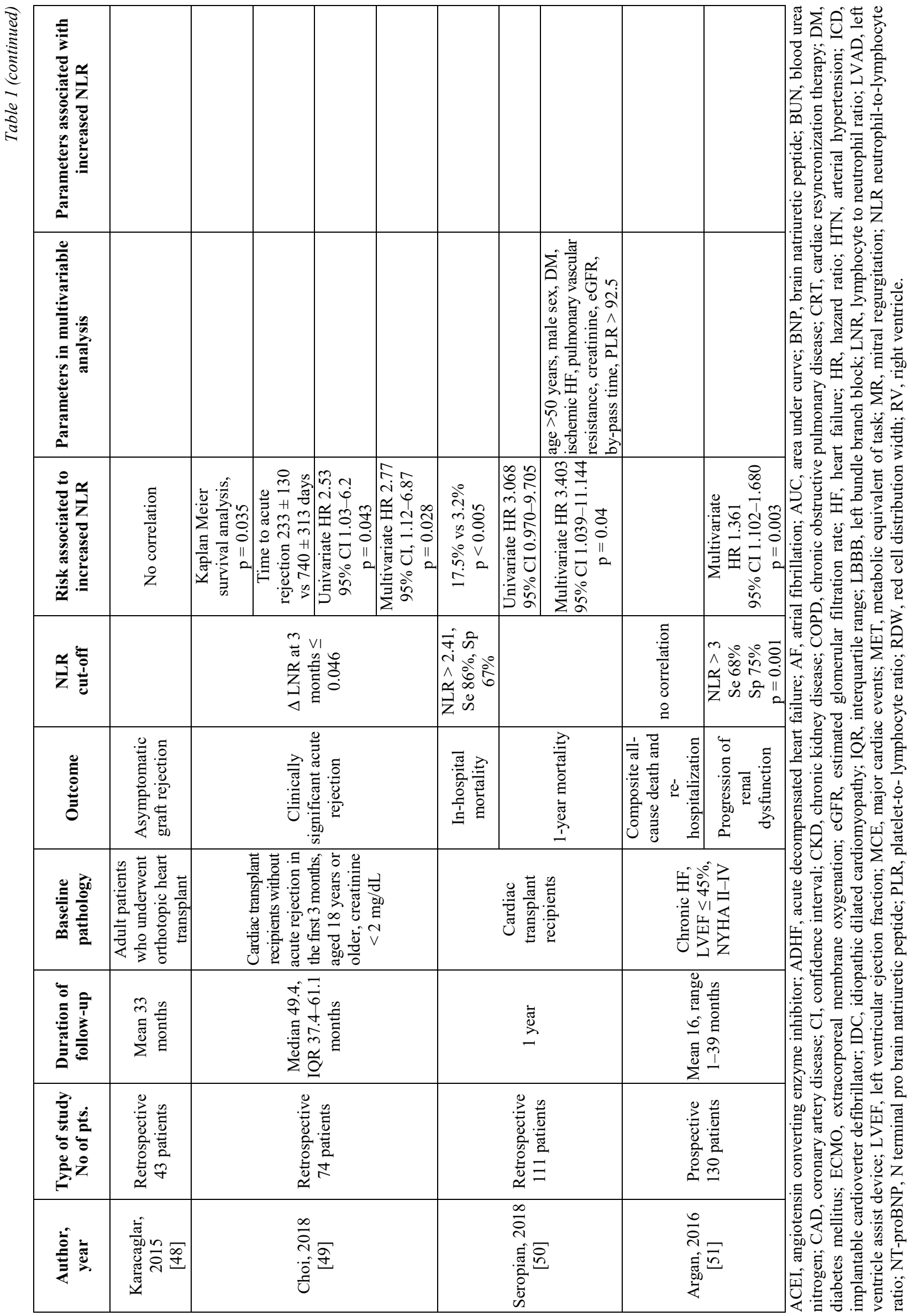




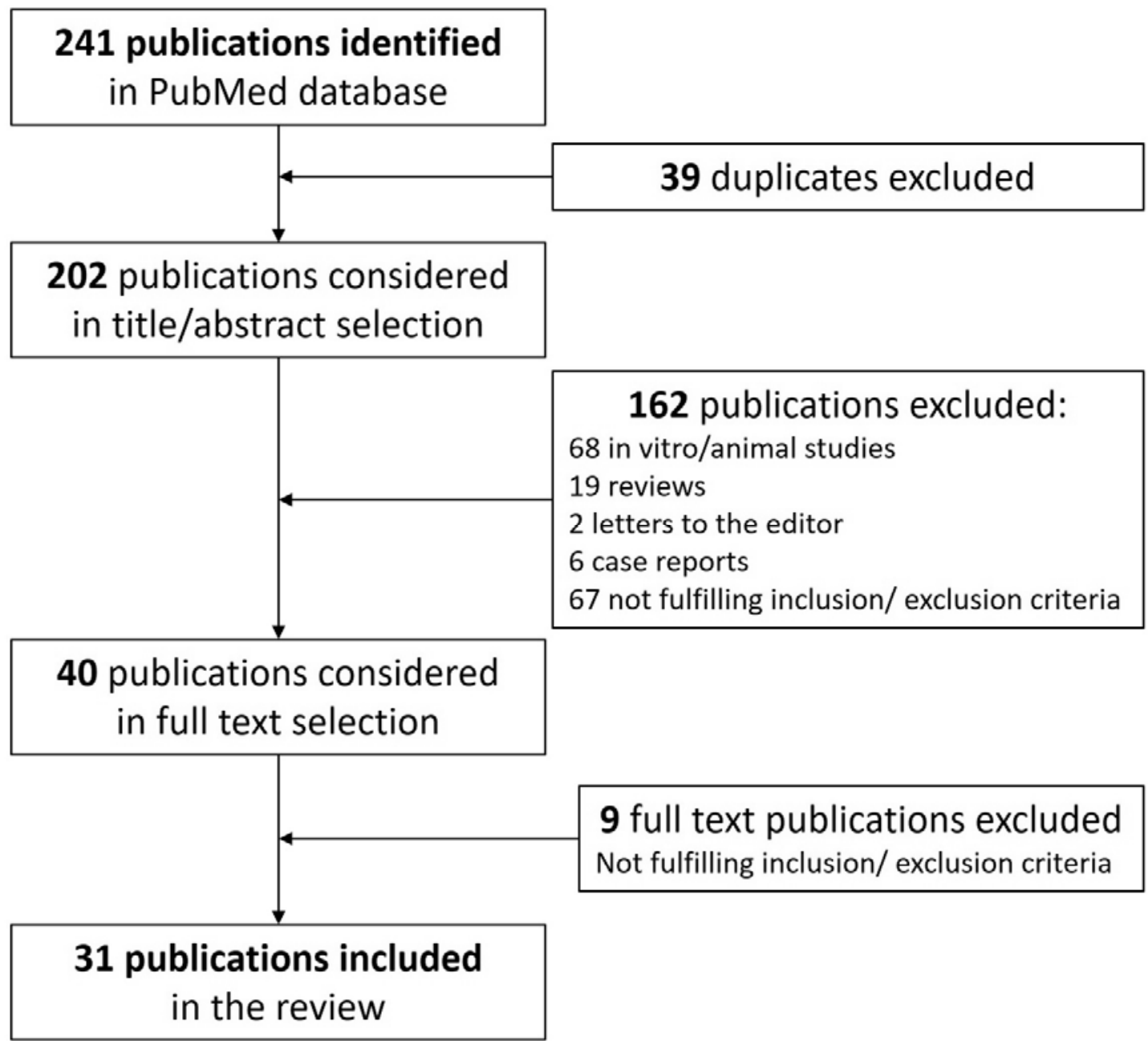

Figure 1. Selection process of articles.

Table 2

Supplemental material. Search strategy

\begin{tabular}{|l|c|}
\hline \multicolumn{1}{|c|}{ Search strategy } & $\begin{array}{c}\text { Retrieved } \\
\text { Articles }\end{array}$ \\
\hline (((neutrophil [Title/Abstract] OR "neutrophils" [MeSH Terms]) AND (lymphocyte [Title/Abstract] OR \\
"lymphocytes" [MeSH Terms])) OR neutrophil lymphocyte [Title/Abstract] OR (("neutrophils" [MeSH \\
Terms] OR "neutrophils" [All Fields] OR "neutrophil" [All Fields]) AND "lymphocytes" [MeSH Terms])) & 112 \\
AND ((heart [Title/Abstract] AND failure [Title/Abstract]) OR "heart failure" [MeSH Terms]) & \\
\hline (((neutrophil [Title/Abstract] OR "neutrophils" [MeSH Terms]) AND (lymphocyte [Title/Abstract] OR & \\
"lymphocytes" [MeSH Terms])) OR neutrophil lymphocyte [Title/Abstract] OR (("neutrophils" [MeSH & 65 \\
Terms] OR "neutrophils" [All Fields] OR "neutrophil" [All Fields]) AND "lymphocytes" [MeSH Terms])) & \\
AND ("cardiomyopathies" [MeSH Terms] OR cardiomyopathy [Title/Abstract]) & \\
\hline (((neutrophil [Title/Abstract] OR "neutrophils" [MeSH Terms]) AND (lymphocyte[Title/Abstract] OR & 53 \\
"lymphocytes" [MeSH Terms])) OR neutrophil lymphocyte [Title/Abstract] OR (("neutrophils" [MeSH & \\
Terms] OR "neutrophils" [All Fields] OR "neutrophil" [All Fields]) AND "lymphocytes" [MeSH Terms])) & \\
AND ((transplant [Title/Abstract] OR transplantation [Title/Abstract]) AND (heart [Title/Abstract] OR "heart & \\
failure" [MeSH Terms])) & \\
\hline ((neutrophil [Title/Abstract] OR "neutrophils" [MeSH Terms]) AND (lymphocyte [Title/Abstract] OR \\
"lymphocytes" [MeSH Terms])) AND (resynchronization [Title/Abstract] OR (resynchronization [All Fields] \\
AND "therapeutics" [MeSH Terms])) & \\
\hline ((neutrophil [Title/Abstract] OR "neutrophils" [MeSH Terms]) AND (lymphocyte [Title/Abstract] OR \\
"lymphocytes" [MeSH Terms])) AND ((implantable [Title/Abstract] AND defibrillator [Title/Abstract] AND \\
(cardiac [Title/Abstract] OR cardioverter [Title/Abstract])) OR ICD [Title/Abstract] OR (implantable [All \\
Fields] AND ("heart" [MeSH Terms] OR "heart" [All Fields] OR "cardiac" [All Fields]) AND "defibrillators" \\
[MeSH Terms]) OR "defibrillators, implantable" [MeSH Terms]) & \\
\hline
\end{tabular}




\section{Congestive heart failure}

\section{General characteristics and heart failure severity}

NLR was correlated to parameters of HF severity, such as New York Heart Association (NYHA) class, left ventricular ejection fraction (LVEF), and natriuretic peptide levels in different groups of HF patients [19, 20, 21]. It was also associated with comorbidities such as atrial fibrillation (AF), arterial hypertension (HTN), diabetes mellitus (DM) and chronic kidney disease (CKD) $[19,20,21]$. Regarding laboratory parameters, higher NLR was correlated to higher creatinine and BUN, lower estimated glomerular filtration rate (eRFG), sodium, albumin, total proteins, and lower hemoglobin levels [19, 20, 21].

In patients with acute decompensated heart failure (ADHF) the highest NLR tertile was associated to older age, female gender, HTN, DM, history of coronary artery disease (CAD), and history of AF [19]. On clinical examination, patients with NLR values in highest tertile had higher heart rates, and higher frequency of pulmonary congestion, peripheral edema, distended jugular veins, as well as higher NYHA class (III or IV) [19]. These patients also had increased incidence of radiological signs of heart failure: cardiomegaly, pleural effusions, and interstitial edema [19]. The highest tertile NLR was also associated with lower LVEF, higher levels of NT-proBNP, BUN, and serum creatinine [19].

In patients with advanced heart failure, higher NLR values were associated with older age, male gender, history of HTN, and the necessity for left ventricular assist device (LVAD) support [20]. These patients also had increased levels of BNP, serum creatinine, BUN and total bilirubin, and decreased levels of serum sodium, albumin, and total proteins [20].

In patients with HF with an LVEF of $35 \%$ or lower, higher NLR levels were correlated to older age, increased prevalence of DM, anemia, AF and CKD stage III and up [21]. Patients from this group with NLR in the highest tertile also had higher NYHA functional class, lower LVEF and more frequently significant valvular disease [21]. From the laboratory parametrs, higher NLR was correlated to lower hemoglobin levels, higher white blood cell count, NT-proBNP, bilirubin, BUN, creatinine and uric acid [21].

\section{Genes associated with elevated NLR in chronic heart failure}

Studying genetic correlations of elevated NLR levels in 197 patients with chronic HF, Wan et al. found associations with interleukin-1 receptor 2 (IL1R2), solute carrier family 22 member 4 (SLC22A4), vanin 3 (VNN3), Annexin A3 (ANXA3), BMX non-receptor tyrosine kinase (BMX), and interleukin-1 receptor type 1 (IL1R1) [22]. These differentially expressed genes were mostly enhanced in neutrophil activation and neutrophil mediated immunity, fluid shear stress and atherosclerosis, as well as transcriptional misregulation in malignancy, suggesting a possible interconnection of these pathways with those activated in heart failure [22]. In multivariate regression analysis including age and gender, a higher expression of SLC22A4 (OR 5.219, $\mathrm{p}=0.003$ ), IL1R2 (OR 5.228, $\mathrm{p}=0.007)$, VNN3 (OR 3.478, $\mathrm{p}=0.012$ ) and male sex were independently associated with elevated NLR in HF patients [22].

\section{Functional capacity in congestive heart failure}

In a small sample of 94 patients with congestive heart failure undergoing symptom-limited treadmill testing to assess functional capacity (FC), NLR was correlated with exercise performance, a marker not only for patients' quality of life, but also a predictor of morbidity and mortality [23]. A cut-off point of 2.74 was established for predicting poor FC [23]. Moreover, patients with NLR $>3$ had more frequent $\mathrm{AF}$, higher NT-proBNP values, increased left atrial (LA) dimensions and systolic pulmonary artery pressure (sPAP), lower LVEF and more severe left ventricular (LV) diastolic dysfunction [23].

\section{In-hospital mortality after acute decompensated heart failure}

Patients with HF admitted for acute decompensation with higher NLR levels had higher in-hospital mortality, as first reported by Uthamalingam et al. Those with NLR values in the highest tertile had an increased rate of in-hospital death, compared to those in the first tertile (OR 2.8, $95 \%$ CI $1.43-5.53, \mathrm{p}=0.03)$ as well as compared to those in the second tertile (OR 1.68, 95\% CI $1.00-3.03, \mathrm{p}=0.05$ ) [19].

Another report of the utility of NLR in HF short-term prognosis is the study authored by Turfan el al., investigating a cohort of 167 patients with acute HF with reduced LVEF $<50 \%$ [24]. NLR predicted in-hospital mortality with an AUC of 0.687 and a cut-off level $>4.78$ with $66.7 \%$ sensitivity and $60.5 \%$ specificity [24]. In multivariate logistic regression, age, LVEF and NLR $>4.78$ (OR 1.156, 95\% CI 1.011-1.334, $\mathrm{p}=0.048$ ) were independent predictors of in-hospital mortality [24]. 
In a large cohort of 638 consecutive patients with acute decompensated heart failure, patients with in-hospital death had significantly higher NLR values compared to those discharged home (3.5 (2.3-5.4) compared to $7.7(4.0-15.3), \mathrm{p}<0.001)$. Higher NLR values were correlated to in-hospital mortality in both univariate and multivariate analysis [25].

Liu et al. also proved that higher NLR values were associated with in-hospital mortality in a sample of 179 patients with chronic failure [26]. Individuals surviving the admission for HF had significantly lower mean NLR levels compared to those that did not $(3.9$ versus $10.2, \mathrm{p}<0.05)$ [26]. NLR was correlated to in-hospital mortality with an AUC of 0.885 (95\% CI 0.799-0.971, p < 0.05) and a cut-off value $>3.317$ with $68.1 \%$ specificity and $100 \%$ sensitivity [26].

Furthermore, NLR proved useful in patients with acute decompensation of advanced heart failure receiving levosimendan treatment, as reported by Tasal et al. [27]. Significant decrease of NLR levels was observed in patients with favorable outcomes after levosimendan infusion [27]. The NLR values after levosimendan treatment were independent predictors of in-hospital mortality with an OR of $1.310,95 \%$ CI $1.158-1.483, p<0.001$, in multivariate analysis including all hemodynamic, hematological, and biochemical variables associated with mortality [27]. In ROC curve analysis a cut-off level of NLR $\geq 5.542$ after levosimendan infusion was established for predicting in-hospital mortality with a $67 \%$ sensitivity and $66 \%$ specificity [27].

\section{Readmissions for acute decompensated heart failure}

Patients evaluated after one admission for acute decompensated heart failure with a high NLR level, respectively in the highest tertile, had a significantly higher 30-day risk of readmission compared to those with NLR values in the second tertile (OR 3.46, 95\% CI 2.11-5.68, $\mathrm{p}<0.001$ ) [19].

\section{Post-discharge mortality after acute decompensated heart failure}

In a large cohort of patients discharged after hospitalization for ADHF, Uthamalingam et al. reported an increased risk of long-term mortality for patients with higher NLR on admission [19]. During a median follow-up period of 26 months (interquartile range 15 to 36 ), patients with NLR values in the highest tertile had an adjusted HR of $2.1,95 \%$ CI $1.53-2.88, \mathrm{p}<0.001$ for mortality compared to those in the first tertile [19]. Compared to those in the second tertile, the adjusted HR was $1.48,95 \%$ CI $1.02-2.14, \mathrm{p}=0.03$ [19].

NLR levels were also associated with increased post-discharge mortality in patients with heart failure evaluated for advanced heart failure therapies and consideration for heart transplant or mechanical circulatory assist devices [20]. After a median follow-up of 11.3 (interquartile range 3.4 to 21.1) months, those with NLR values in the second (adjusted HR 2.16, 95\% CI 1.21-3.83, p < 0.001) and third tertiles (adjusted HR 1.83, 95\% CI 1.07-3.14, $\mathrm{p}=0.03$ ) had significantly higher risk of all-cause mortality, compared to the first tertile [20].

After a mean follow-up period of $12.8 \pm 7.6$ months in small cohort of $56 \mathrm{HF}$ patients with both reduced as well as preserved EF, NLR predicted all-cause mortality with an AUC of 0.730 and a cut-off level of NLR $>5.1$, with $75 \%$ sensitivity and $62 \%$ specificity $(\mathrm{p}=0.045)$ [28]. In multivariate logistical regression alongside age, sex, LVEF and platelet to lymphocyte ratio, NLR was an independent predictor of mortality with an OR of 1.680 (95\% CI $1.013-2.786, \mathrm{p}=0.045)$ [28].

NLR was also an independent predictor of all-cause mortality (adjusted HR 1.039, 95\% CI $1.007-1.073, \mathrm{p}=0.016)$ in a cohort of $306 \mathrm{HF}$ patients aged over 60 years, followed-up for a mean period of 471 (median 342) days, alongside age, sex, plasma albumin, and NT-proBNP levels [29].

Patients with HF with a LVEF $\leq 35 \%$ with NLR values in the highest tertile had a HR of 1.43 $(95 \%$ CI $1.1-1.85, p=0.006)$ of all-cause mortality during a median follow-up period of 660 days (interquartile range, 331-1074 days) [21]. During the first 12 months after inclusion, in the entire study group, an increase in mortality of almost 3 -fold was observed for patients with the highest tertile NLR values compated to those in the lowest tertile [21]. After adjusting for other predictors of survival, in multivariate analysis, NLR levels in the highest tertile were predictors of long-term mortality only for patients with ischemic HF (HR $1.51,95 \%$ CI $1.11-2.04, \mathrm{p}=0.008)$, and not for those with non-ischemic HF (HR of $1.22,95 \%$ CI $0.77-1.94, \mathrm{p}=0.39)$ [21].

Huang et al. also reported the role of NLR for post-discharge mortality prediction in 1923 patients hospitalized for ADHF followed-up for a mean duration of $28.6 \pm 20.7$ months [30]. NLR was a significant predictor of mortality in univariate analysis (HR 1.160, 95\% CI 1.112-1.210, p < 0.01) as well as multivariate analysis adjusting for NT-proBNP levels 
(HR 1.137, 95\% CI 1.015-1.274, $\mathrm{p}=0.03$ ) or in multivariate analysis adjusting for age, sex, mean blood pressure, LVEF, serum sodium levels, hemoglobin, eGFR, treatment with renin-angiotensin system blockers, beta-blockers and spironolactone (HR 1.162, 95\% CI 1.094-1.235, p < 0.01) [30].

\section{Special populations of heart failure}

\section{Dilated cardiomyopathy}

In patients with idiopathic dilated cardiomyopathy (IDC), higher NLR values were associated to higher NYHA class and higher BNP levels [31]. NLR was correlated to the severity of chronic HF, a cut-off value of NLR $\geq 2.25$ associating $82 \%$ sensitivity and $65 \%$ specificity to predict severity of the cardiac condition[31]. Parameters of cardiac remodeling, such as LV and LA volumes and dimensions, LV systolic and diastolic function, sPAP and right ventricular systolic motion were significantly impaired in patients with a NLR $\geq 2.25$ [31].

$\mathrm{FC}$ is an important prognostic predictor in heart failure. In patients with IDC, NLR was significantly correlated to FC assessed by treadmill testing, correlating therefore with their outcomes [32]. In the same cohort, NLR was also correlated to both systolic and diastolic LV dysfunction, independently of the FC [32].

However, regarding prognosis, during a five-year follow-up of a small HF group with advanced idiopathic or familial dilated cardiomyopathy evaluated for non-urgent heart transplant, SobrinoMarquez et al. found no correlation of median NLR on initial work-up with survival or necessity for surgical intervention [33].

\section{Heart failure in elderly patients}

In elderly patients with CHF, NLR was independently correlated with the occurrence of major cardiovascular events (MCE), as well as with CKD [34]. During a median follow-up period of 18 months, those with the highest tertile of the NLR (median 4.1, interquartile range 3.39 to 6.01 ) had a significantly higher incidence of cardiac death and rehospitalization for heart failure [34].

Studying the same cohort of 1355 older patients, Yan $\mathrm{W}$ et al. also found an independent correlation between NLR and the presence of AF [35]. Moreover, when computing the multivariate analysis including the NT-proBNP values, NLR was an independent predictor of MCE [35]. Patients with the NLR levels in the highest tertile had a 1.407-fold higher risk of MCE [35].

\section{Peripartum cardiomyopathy}

Given the hypothesis of systemic inflammation contributing to the pathophysiology of peripartum cardiomyopathy, biomarkers such as C-reactive protein, TNF- $\alpha$, and interleukin- 6 were correlated to severity and prognosis of this disease [36, 37]. Gurkan U. and his collaborators also proved the utility of NLR, as an inflammatory biomarker, in 40 patients with peripartum cardiomyopathy [38]. In multivariate logistic regression, NLR was identified as an independent predictor of persistent LV systolic dysfunction after a mean follow-up period of $34.8 \pm$ 23 months [38].

\section{Response to device therapy in heart failure}

\section{Resynchronization therapy}

Studying a cohort of 70 patients undergoing cardiac resynchronization therapy (CRT) implantation, Agacdiken et al. proved the role of baseline NLR in predicting clinical and functional response to the device therapy [39]. In multivariable analysis including age, etiology of cardiomyopathy, baseline LVEF, NYHA functional class, CRP, and NLR, NLR was the only independent predictor of CRT responsiveness at 6 months (OR 1.506, 95\% CI 1.011-2.243, $\mathrm{p}=0.035$ ) [39]. Moreover, a significant decrease in mean NLR values from $2.4 \pm 1$ at baseline to $2.1 \pm 0.7$ after 6 months was associated with echocardiographic response to CRT [39].

Balci et al. also analyzed a group of 157 patients with severe symptomatic HF despite optimal medical therapy, with reduced LVEF and left bundle branch block that received resynchronization therapy in combination with a cardioverter defibrillator [40]. NLR levels $>3.45$ before CRT implantation predicted non-responsiveness to the therapy with a $64 \%$ sensitivity and $74.8 \%$ specificity and an AUC in ROC analysis of $0.718(95 \%$ CI $0.633-0.803, \mathrm{p}<0.001)$ [40]. In multivariate analysis alongside ischemic etiology, prior percutaneous coronary intervention (PCI) reperfusion therapy, history of HTN, and statin treatment, NLR $>3.45$ was an independent predictor of CRT non-responsiveness (OR 12.216, 95\% CI 2.161-69.052, $\mathrm{p}=0.005)$ [40].

The utility of NLR in predicting response to CRT was also confirmed in a prospective study by Boros and collaborators [41]. Patients with lower baseline NLR achieved reverse remodeling of the left ventricle after 6 months post CRT implantation (OR 0.38, 95\% CI 0.17-0.85, $\mathrm{p}=0.01$ for NLR $\leq 2.95$ ) [41]. Patients with higher baseline NLR $(>2.95)$ had a higher risk for all-cause mortality during the 
follow-up period of two years (HR 2.44, 95\% CI 1.04-5.71, $\mathrm{p}=0.03$ ) [41]. Both statistical analysis for the prediction of reverse remodeling and all-cause long-term mortality were computed using multiple prediction parameters, including NT-proBNP values, making NLR an independent predictor of outcomes [41].

\section{Implantable cardioverter defibrillator}

In 120 patients with heart failure NYHA classes II and III, with implantable cardioverter defibrillators (ICD) for either primary or secondary prevention, higher NLR was correlated to all-cause mortality but not to appropriate ICD shocks [42]. In multivariate survival analysis, NLR, BNP levels and eGFR were independent predictors of mortality during a median follow-up period of 61.2 months [42]. Patients with lower NLR levels $(<2.1)$ had decreased mortality, but not different numbers of appropriate ICD shocks [42].

In patients with IDC receiving an ICD for primary prevention of sudden cardiac death, NLR did predict arrhythmic events and appropriate ICD therapy with an AUC of 0.707 (95\% CI 0.629-0.785, $\mathrm{p}<0.001$ ), higher NLR being correlated to these outcomes [43]. Consequently, Ucar et al. suggested the use of NLR as an additional biomarker for stratifying the ventricular arrhythmic risk in patients with IDC [43].

\section{Left ventricular assist device}

In a cohort of 273 patients with advanced HF who received left ventricular assist devices (LVAD), NLR was an independent predictor of postoperative mortality (OR 1.159, 95\% CI 1.022-1.314, p = 0.021) as well as postoperative right ventricular failure (OR 1.117, 95\% CI 1.039-1.201, p = 0.003) [44]. Associated with older age, ischemic etiology of $\mathrm{HF}, \mathrm{CKD}$ and increased incidence of ventricular tachycardia, NLR was also directly correlated to length of hospital stay [44].

Bhat et al. further proved that the NLR variability in time was correlated to outcomes in HF patients receiving LVAD therapy [45]. Highest NLR levels were recorded immediately after surgery with a tendency to decrease after approximately 30 days to levels similar to the preoperative ones [45]. However, patients whose NLR failed to improve 10 days postoperative had higher probability of a longer length of stay, of developing right $\mathrm{HF}$ and a trend toward reduced survival rates [45].

Another longitudinal analysis of NLR in patients with advanced HF receiving LVAD therapy as bridge to transplant, carried out by Ferrera et al., confirmed the rise and fall in NLR levels immediately after and several months after surgery [46]. Patients with NLR higher than median during the 4-6 months follow-up had higher rates of stroke and mortality [46]. NLR during follow-up higher than 4.4 was an independent predictor of mortality, associating significantly shorter survival time [46].

Retrospectively examining a cohort of 301 patients with LVAD support monitored for 365 days, Sundararajan et al. highlighted the importance of determining NLR levels both pre-LVAD and during follow-up [47]. Higher NLR prior to LVAD implantation as well as higher NLR after 90 days post-operatively were independent predictors of mortality [47].

\section{Heart transplantation}

In patients with advanced HF during a median follow-up period of 11.3 months (IQR 3.4 to 21.1), higher NLR values did not correlate to more frequent heart transplantation (adjusted HR 0.80, 95\% CI $0.47-1.43, \mathrm{p}=0.43)$ [20].

In a retrospective analysis of 47 patients who received orthotopic heart transplant, periodically followed-up with routine endomyocardial biopsies, Karacaglar et al. found no correlation between NLR and to asymptomatic graft rejection [48]. This study measured NLR on each visit and correlations were made to the results of EMB collected on the same evaluation.

However, when evaluating lymphocyte to neutrophil ratio (LNR) variability in time, at baseline, 3, 6 and 12 months after heart transplantation in a cohort of 74 patients, decreasing LNR was correlated with acute rejection [49]. Lower LNR during the first three months of follow-up was an independent predictor of clinically overt acute graft rejection [49]. In other words, higher NLR would be associated with acute rejection after heart transplantation.

Another study published by Seropian et al. proved that higher NLR before heart transplantation was an independent predictor of one-year mortality (HR 3.403, p < 0.05) [50]. Higher NLR on admission was correlated to higher necessity of renal replacement therapy $(\mathrm{p}<0.05)$ and trending towards an association with higher in-hospital mortality (AUC $0.644,95 \%$ CI 0.492-0.797) [50].

\section{Heart failure and renal dysfunction}

Monitoring the renal function of patients with HF with reduced LVEF during a 16-month follow-up 
period, Argan et al. observed that NLR levels were correlated to kidney disease progression [51]. In ROC analysis, NLR $>3$ had a sensitivity of $68 \%$ and specificity of $75 \%$ to predict worsening renal function with an AUC of $0.72,95 \%$ CI $0.58-0.85$, $\mathrm{p}=0.001$ [51]. Furthermore, patients with a NLR $<3$ had significantly higher rates of survival without progression of kidney dysfunction [51].

\section{CONCLUSIONS}

In HF patients, NLR was correlated to severity of the cardiac condition quantified by higher NYHA class and NT-proBNP levels, and lower LVEF. NLR was increased in patients with higher comorbidity burden, including valvular heart disease, ischemic coronary disease, AF, DM, HTN, and CKD.
Factors generally associated with poor prognosis in HF, such as older age, increased levels of creatinine, BUN, uric acid, bilirubin, liver enzymes and decreased levels of hemoglobin, albumin, and sodium were associated with higher NLR levels. These patients also presented with echocardiographic parameters of advancing HF, including LV systolic and/or diastolic dysfunction and increased pulmonary artery systolic pressure.

NLR was an independent predictor of all-cause mortality in numerous studies, in various subcategories of HF patients ranging from stable to end-stage, and from indications of device implantation to cardiac transplant, with a cut-off level ranging from $>2.1$ to $>7.6$.

Therefore, in HF patients, increased NLR could be a valuable auxiliary biomarker of severity, but most of all, of poor prognosis.

Introducere. Insuficiența cardiacă (IC) și inflamația sistemică sunt procese interdependente ce se potențează reciproc, continuu. Diverse căi fiziopatologice sunt activate, rezultând în creșterea numărului de neutrofile și scăderea numărului de limfocite, ceea ce face din raportul neutrofile-limfocite (NLR) un potențial marker indirect de severitate. Am realizat această lucrare pentru a caracteriza rolul NLR in $I C$.

Metode. Folosind cuvintele cheie "neutrophil", "lymphocyte", "heart failure", "cardiomyopathy", "implantable cardioverter defibrillator", "cardiac resynchronization therapy" și "heart transplant" am căutat articole in baza de date PubMed (MEDLINE).

Rezultate. Am indentificat 241 publicații. 31 au fost selectate pentru această sinteză, incluzând 12107 pacienți. NLR a fost corelat cu severitatea IC exprimată prin parametri clinici, biologici și imagistici, precum și cu prognosticul pe termen scurt și lung. Majoritatea studiilor au raportat valoarea lui prognostică pentru supraviețire. $N L R$ crescut $(>2.1-7.6)$ a fost predictor independent pentru mortalitatea intra-spital [HR ajustat 1.13(95\% CI 1.01-1.27)-2.8(95\% CI 1.43-5.53)] precum și pentru mortalitatea de orice cauză pe termen lung [HR ajustat 1.43(95\% CI 1.1-1.85)2.403(95\% CI 1.076-5.704)].

Valorile mari ale NLR au fost de asemenea predictori pentru capacitatea funcțională scăzută $[N L R>2.26 / 2.74$, HR $3.93(95 \%$ CI 1.02-15.12)/3.085 (95\% CI 1.52-6.26)], reinternări [NLR > 2.9/7.6, HR 1.46 (95\% CI 1.10-1.93)/3.46 (95\% CI 2.11-5.68)], terapia de resincronizare eficientă $(N L R>3.45 /$ creșterea cu o unitate, HR 12.22 (95\% CI 2.16-69.05)/1.51 (95\% CI 1.01-2.24)] şi administrarea de șocuri adecvate prin defibrilatorul implantabil (NLR $>2.93)$, precum și mortalitatea după implantarea dispozitivului de asistare ventriculară [NLR>4.4/cuartile, HR 1.67 (95\% CI 1.03-2.70)/1.22 (95\% CI 1.01-1.47)] sau după transplantul cardiac (NLR > 2.41, HR 3.403 (95\% CI 1.04-11.14)].

Concluzie. NLR crescut la pacienții cu IC poate fi un biomarker auxiliar valoros pentru cuantificarea severității și, mai ales, pentru prognostic.

Correspondence to: Cătălin Adrian Buzea MD, PhD, Cardiology Department Spitalul Clinic Colentina, 19-21 Ștefan cel Mare, Pavilion E, Sector 2, București, România

E-mail: adrian_c_buzea@yahoo.com

Conflict of interest disclosure: The authors have no conflicts of interest to declare. 


\section{REFERENCES}

1. SHIRAZI, L.F., BISSETT, J., ROMEO, F., MEHTA, J.L. Role of inflammation in heart failure. Current Atherosclerosis Reports. 2017; 19(6):27.

2. VAN LINTHOUT, S., TSCHÖPE, C. Inflammation - cause or consequence of heart failure or both?. Curr. Heart Fail. Rep. 2017; 14(4):251-265.

3. SETA, Y., SHAN, K., BOZKURT, B., ORAL, H., MANN, D.L. Basic mechanisms in heart failure: the cytokine hypothesis. J. Card. Fail. 1996; 2(3):243-9.

4. JAHNG, J.W.S., SONG, E., SWEENEY, G. Crosstalk between the heart and peripheral organs in heart failure. Exp. Mol. Med. 2016; 48(3):e217.

5. HARTUPEE, J., MANN, D.L. Positioning of inflammatory biomarkers in the heart failure landscape. J. Cardiovasc. Transl. Res. $2013 ; 6(4): 485-92$.

6. TRACCHI, I., GHigliotTI, G., MURA, M., GARIBALDI, S., SPALlAROSSA, P., BARISIONE, C., et al. Increased neutrophil lifespan in patients with congestive heart failure. Eur. J. Heart Fail. 2009; 11(4):378-385.

7. RUDOLPH, V., RUDOLPH, T.K., HENNINGS, J.C., BLANKENBERG, S., SCHNABEL, R., STEVEN, D., et al. Activation of polymorphonuclear neutrophils in patients with impaired left ventricular function. Free Radic. Biol. Med. 2007; 43(8):1189-1196.

8. ARRUDA-OLSON, A.M., REEDER, G.S., BELL, M.R., WESTON, S.A., ROGER, V.L. Neutrophilia predicts death and heart failure after myocardial infarction: A community based study. Circ Cardiovasc Qual Outcomes 2009; 2(6):656-662.

9. SHAH, A.D., DENAXAS, S., NICHOLAS, O., HINGORANI, A.D., HEMINGWAY, H. Neutrophil counts and initial presentation of 12 cardiovascular diseases. JACC 2017; 69(9):1160-9.

10. MAISEL, A.S., KNOWLTON, K.U., FOWLER, P., REARDEN, A., ZIEGLER, M.G., MOTULSKY, H.J., et al. Adrenergic control of circulating lymphocyte subpopulations. Effects of congestive heart failure, dynamic exercise, and terbutaline treatment. J. Clin. Invest. 1990; 85(2):462-7.

11. VADUGANATHAN, M., GREENE, S.J., BUTLER, J., SABBAH, H.N., SHANTSILA, E., LIP, G.Y.H., et al. The immunological axis in heart failure: Importance of the leukocyte differential. Heart Fail. Rev. 2013; 18(6):835-845.

12. YUCEL, H., EGE, M.R., ZORLU, A., KAYA, H., BETON, O., GUNGOR, H., et al. Lymphocytopenia is associated with poor NYHA functional class in chronic heart failure patients with reduced ejection fraction. Turk Kardiyol. Dern. Arsivi-Archives Turkish Soc. Cardiol. 2015; 43(5):427-433.

13. CHARACH, G., GROSSKOPF, I., ROTH, A., AFEK, A., WEXLER, D., SHEPS, D., et al. Usefulness of total lymphocyte count as predictor of outcome in patients with chronic heart failure. Am. J. Cardiol. 2011; 107(9):1353-1356.

14. VADUGANATHAN, M., AMBROSY, A.P., GREENE, S.J., MENTZ, R.J., SUBACIUS, H.P., MAGGIONI, A.P., et al. Predictive value of low relative lymphocyte count in patients hospitalized for heart failure with reduced ejection fraction. Circ. Hear. Fail. 2012; 5(6):750-758.

15. BHAT, T., TELI, S., RIJAL, J., BHAT, H., RAZA, M., KHOUEIRY, G., et al. Neutrophil to lymphocyte ratio and cardiovascular diseases: a review. Expert Rev. Cardiovasc. Ther. 2013; 11(1):55-9.

16. ZHANG, S., DIAO, J., QI, C., JIN, J., LI, L., GAO, X., et al. Predictive value of neutrophil to lymphocyte ratio in patients with acute ST segment elevation myocardial infarction after percutaneous coronary intervention: a meta-analysis. BMC Cardiovasc. Disord. 2018; 18(1):75.

17. PAQUISSI, F.C. The predictive role of inflammatory biomarkers in atrial fibrillation as seen through neutrophil-lymphocyte ratio mirror. J. Biomarkers 2016; 2016:1-14.

18. AFARI, M.E., BHAT, T. Neutrophil to lymphocyte ratio (NLR) and cardiovascular diseases: an update. Expert Rev. Cardiovasc. Ther. 2016; 14(5):573-577.

19. UTHAMALINGAM, S., PATVARDHAN, E. A, SUBRAMANIAN, S., AHMED, W., MARTIN, W., DALEY, M., et al. Utility of the neutrophil to lymphocyte ratio in predicting long-term outcomes in acute decompensated heart failure. Am. J. Cardiol. 2011; 107(3):433-438.

20. BENITES-ZAPATA, V. A., HERNANDEZ, A. V., NAGARAJAN, V., CAUTHEN, C. A., STARLING, R.C., WILSON TANG, W.H. Usefulness of neutrophil-to-lymphocyte ratio in risk stratification of patients with advanced heart failure. Am. J. Cardiol. 2015; 115(1):57-61.

21. WASILEWSKI, J., PYKA, Ł., HAWRANEK, M., OSADNIK, T., KUREK, A., SKRZYPEK, M., et al. Prognostic value of neutrophil-to-lymphocyte ratio in predicting long-term mortality in patients with ischemic and nonischemic heart failure. Pol. Arch. Med. Wewn. 2016; 126(3):166-173.

22. WAN, G., JI, L., XIA, W., CHENG, L., ZHANG, Y. Screening genes associated with elevated neutrophil-to-lymphocyte ratio in chronic heart failure. Mol. Med. Rep. 2018; 18(2):1415-1422.

23. CAKICI, M., CETIN, M., DOGAN, A., OYLUMLU, M., POLAT, M., SUNER, A. Neutrophil to lymphocyte ratio predicts poor functional capacity in patients with heart failure. Arch Turk Soc Cardiol 2014; 42(7):612-620.

24. TURFAN, M., ERDOGAN, E., TASAL, A., VATANKULU, M., JAFAROV, P., SONMEZ, O., et al. Neutrophil-to-lymphocyte ratio and in-hospital mortality in patients with acute heart failure. Clinics 2014; 69(3):190-193.

25. OSTROWSKA, M., OSTROWSKI, A., ŁUCZAK, M., JAGUSZEWSKI, M., ADAMSKI, P., BELLWON, J., et al. Basic laboratory parameters as predictors of in-hospital death in patients with acute decompensated heart failure: data from a large single-centre cohort. Kardiol. Pol. 2016; 75(2):157-163.

26. LIU, S., WANG, P., SHEN, P.-P., ZHOU, J.-H. Predictive values of red blood cell distribution width in assessing severity of chronic heart failure. Med. Sci. Monit. 2016; 22:2119-2125.

27. TASAL, A., ERTURK, M., UYAREL, H., KARAKURT, H., BACAKSIZ, A., VATANKULU, M.A., et al. Utility of the neutrophil to lymphocyte ratio for predicting in-hospital mortality after levosimendan infusion in patients with acute decompensated heart failure. J. Cardiol. 2014; 63(6):418-423. 
28. DURMUS, E., KIVRAK, T., GERIN, F., SUNBUL, M., SARI, I., ERDOGAN, O. Neutrophil-to-lymphocyte ratio and plateletto-lymphocyte ratio are predictors of heart failure. Arq. Bras. Cardiol. 2015; (June 2012):606-613.

29. FU, S., XIE, L., LI, D., YE, P., LUO, L. The predictive capacity and additional prognostic power of N-terminal pro-B-type natriuretic peptide in Chinese elderly with chronic heart failure. Clin. Interv. Aging 2015; 10:359-365.

30. HUANG, W.M., CHENG, H.M., HUANG, C.J., GUO, C.Y., LU, D.Y., LEE, C.W., et al. Hemographic indices are associated with mortality in acute heart failure /692/4019/592/75/74/692/4019/592/75/230 article. Sci. Rep. 2017; 7(1):1-9.

31. AVCI, A., ALIZADE, E., FIDAN, S., YESIN, M., GULER, Y. Neutrophil/lymphocyte ratio is related to the severity of idiopathic dilated cardiomyopathy. Scand Cardiovasc J 2014; 48(4):202-8.

32. YILDIZ, A., YÜKSEL, M., OYLUMLU, M., POLAT, N., AKIL, M.A., ACET, H. The association between the neutrophil/ lymphocyte ratio and functional capacity in patients with idiopathic dilated cardiomyopathy. Anadolu Kardiyol. Derg. 2015; 15(1):13-17.

33. SOBRINO-MÁRQUEZ, J.M., GRANDE-TRILLO, A., CANTERO-PÉREZ, E.M., RANGEL-SOUSA, D., LAGE-GALLE, E., ADSUAR-GÓMEZ, A. Prognostic value of blood panel parameters in patients with dilated cardiomyopathy and advanced heart failure. Transplant. Proc. 2018; 50(2):650-652.

34. YAN, W., LIU, C., LI, R., MU, Y., JIA, Q., HE, K. Usefulness of the neutrophil-to-lymphocyte ratio in predicting adverse events in elderly patients with chronic heart failure. Int. Heart J. 2016; 57(5):615-621.

35. YAN, W., LI, R.J., JIA, Q., MU, Y., LIU, C.L., HE, K.L. Neutrophil-to-lymphocyte ratio compared to N-terminal pro-brain natriuretic peptide as a prognostic marker of adverse events in elderly patients with chronic heart failure. J. Geriatr. Cardiol. 2017; 14(2):127-134.

36. SAROJINI, A., SAI RAVI SHANKER, A., ANITHA, M. Inflammatory markers-serum level of c-reactive protein, tumor necrotic factor- $\alpha$, and interleukin- 6 as predictors of outcome for peripartum cardiomyopathy. J. Obstet. Gynecol. India 2013; 63(4):234-239.

37. AZIBANI, F., SLIWA, K. Peripartum cardiomyopathy: an update. Curr. Heart Fail. Rep. 2018; 15(5):297-306.

38. GÜRKAN, U., AKGÖZ, H., AKSOY, Ş., CAN GÜRKAN, Ö., OSKEN, A., UNAL DAYI, S., et al. Value of the neutrophil-tolymphocyte ratio in predicting left ventricular recovery in patients with peripartum cardiomyopathy. Wien. Klin. Wochenschr. 2017; 129(23-24):893-899.

39. URAL, D. Neutrophil-to-lymphocyte ratio predicts response to cardiac resynchronization therapy. Med. Sci. Monit. 2013; 19:373-377.

40. BALCI, K.G., BALCI, M.M., SEN, F., CANPOLAT, U., AKBOGA, M.K., UNAL, S., et al. The role of baseline indirect inflammatory markers in prediction of response to cardiac resynchronisation therapy. Kardiol. Pol. 2016; 74(2):119-126.

41. BOROS, A.M., SZÉPLAKI, G., PERGE, P., JENEI, Z., BAGYURA, Z., ZIMA, E., et al. The ratio of the neutrophil leucocytes to the lymphocytes predicts the outcome after cardiac resynchronization therapy. Europace 2016; 18(5):747-754.

42. HASHIMOTO, N., ARIMOTO, T., NARUMI, T., IWAYAMA, T., KUTSUZAWA, D., ISHIGAKI, D., et al. The neutrophil-tolymphocyte ratio predicts all-cause mortality in patients with implantable cardioverter defibrillators. PACE - Pacing Clin. Electrophysiol. 2017; 40(2):135-144.

43. UCAR, F., ACAR, B. Neutrophil to lymphocyte ratio predicts appropriate therapy in idiopathic dilated cardiomyopathy patients with primary prevention implantable cardioverter defibrillator. Saudi Med J 2017; 38(2):143-148.

44. YOST, G., JOSEPH, C., TATOOLES, A., BHAT, G. Neutrophil-to-lymphocyte ratio predicts outcomes in patients implanted with left ventricular assist devices. ASAIO J. 2015; 61:664-9.

45. BHAT, G., YOST, G.L., IBRAHIM, K., PAPPAS, P., TATOOLES, A. Risk stratification with longitudinal neutrophil to lymphocyte ratio assessment after left ventricular assist device implantation. Int. J. Artif. Organs 2018; 41(8):445-451.

46. FERRERA, C., GONZALEZ FERNANDEZ, O., BOUZAS, N., CASTRODEZA, J., GREEN, T., WOODS, A., et al. Neutrophil to lymphocyte ratio is related to thrombotic complications and survival in continuous flow left ventricular assist devices. ASAIO J. 2019; [Epub ahead of print]

47. SUNDARARAJAN, S., KIERNAN, M.S., COUPER, G.S., UPSHAW, J.N., DENOFRIO, D., VEST, A.R. The neutrophillymphocyte ratio and survival during left ventricular assist device support. J. Card. Fail. 2019; 25(3):188-194.

48. KARACAGLAR, E., BAL, U., CIFTCI, O., TURGAY, O., YILMAZ, M., SADE, E., et al. Predictive value of hematologic parameters for detecting asymptomatic graft rejection after heart transplant: Preliminary results. Exp. Clin. Transplant. 2015; 13:146-148.

49. CHOI, D.-H., KOBAYASHI, Y., NISHI, T., LUIKART, H., DIMBIL, S., KOBASHIGAWA, J., et al. Change in lymphocyte to neutrophil ratio predicts acute rejection after heart transplantation. Int. J. Cardiol. 2018; 251:58-64.

50. SEROPIAN, I.M., ROMEO, F.J., PIZARRO, R., VULCANO, N.O., POSATINI, R.A., MARENCHINO, R.G., et al. Neutrophilto-lymphocyte ratio and platelet-to-lymphocyte ratio as predictors of survival after heart transplantation. ESC Hear. Fail. 2018; 5(1):149-156.

51. ARGAN, O., URAL, D., KOZDAG, G., SAHIN, T., BOZYEL, S., AKTAS, M., et al. Associations between neutrophil gelatinase associated lipocalin, neutrophil-to-lymphocyte ratio, atrial fibrillation and renal dysfunction in chronic heart failure 2016; 4765-4772.

Received June $1^{\text {st }} 2019$ 